\title{
Síndrome Fetal Alcohólico. Estudio de 19 casos clínicos
}

Dr.: Maria Mena R., Carnen Albornoz V.,"

Sras,: Mark C. Puente ${ }^{* *}$, Cecilia Moreno" ${ }^{m * *}$

\begin{abstract}
The authors reports 19 infants of Alcoholic Mathers. The aroup comprised 7 families.

A clinical and laboralory check up was performed: hemogrom, urine $X$ ray of skull and wrist, dermatoglyphys and intellectual developsnent.

The temlonenetic effect of alcohol was dyemorphorenesis which raried according to amount and frequency of alcohol ingested. Besides, it depended on age of pregnacy.

Morphogenic alterations of S.F.A. were in direct proportion to the psychomotor and intellectual retordation.

The severity of S.F.A. was greater in direct correlation with the duration of meternal alcoholism.

The inteasity of maternal or fanilial alcoholism was directly proportional to infantile undernourishment.

Obstetrical charts of alcoholic mothera revealed increase of spontaneous abortions, prenature childbirth, atillbirths and infont mortality.
\end{abstract}

Desde hace muchos años se sospechaba que el alcohol ingerido por la madre durante el embarazo, tenúa efectos teratogénicos para el feto.

Así, en 1834, House y Commons observaron que los hijos de madres alcohólicas tenían un estado nutritivo insuficiente y alteraciones morfológicas de los ojos.

En 1900, Sullivan encontró alta incidencia de mortinatos en hüjos de mujeres alcohólicas y, al año siguiente, Lechange comunicaba que los hjjos de atcohólicas eran pequeños y enfermizos.

En la literatura médica francesa, Lemoine, en 1968, reporto que existía un desarrollo morfológico y fetal anómalo, además de una deficiencia importante en la función intelectual. ${ }^{19}$

Jones y Smith, en 1973 , estudiaron ampliamente las manifestaciones morfológicas más importantes y frecuentes encontradas en el S.F.A., destacando los principales aspectos para un reconocimiento precoz. ${ }^{7,8}$

Los principales rasgos de S.F.A. son:

1. Trastomos del S.N.C., retardo mental, incoordinación motora, hiperactividad, hipotonía.

2. Trastornos en el desarrollo prenatal y postnatal: menor que 2 D.S.

3. Trastornos cráneo-faciales: microcefalia, mi-

* Servicio de Pediatria Hospital Bane Valdivis e Inotituto de Pediatria, Universidad Austral de Chile.

+*Instituto de Psiquialria, Univeraidad Angtral do Chile.

***Policlínico de Desnutridos, Hospital Bage Valdivia. crooftalmia, ptosis palpebral, hendiduras palpebrales cortas, estrabismo, miopía, hipoplasia medio facial, hipoplasia maxilar, prognatiamo relativo, paladar asimétrico, paladar ojival, orejas posteriores.

4. Malformaciones variables: cardiopatía congénita, líneas palmares aberrantes, hirsutismo, etc. Según Sterling ${ }^{14}$ todos los pacientes con S.F.A. han procedido de mujerea alcohólicas que consumen diariamente dosis elevadas de alcohol, o lo ingieren en forma frecuente. El consumo diario de $89 \mathrm{cc}$. de alcohol puro, equivalente a tres onzas, constituyen el mayor riesgo para el feto. Se desconoce el efecto teratógeno cuando la ingestión y frecuencia son menores a las señaladas y se deduce que el efecto es menor.

Como todo teratógeno las manifestaciones que provoca son variables. En el grado más severo del espectro están los pacientes con la constelación de anomalías del S.F.A., presentíndose también grados moderados y leves del sindrome.

Estudios realizados por distintos autores indican que el riesgo fetal existente para los hijos de madre alcohólica, es de 30-50\%.2, 5. o

Un gran número de casos clínicos de S.F.A., han sido reportados en la literatura médica de EE.UU. y Europa y refieren que la frecuencia actual del síndrome es de 1 a $2 / 1000$ recién nacidos y con expresión parcial de 3 a $5 / 1000$. $^{14}$

En nuestro Servicio el hecho de constatar que en varias familias de desnutridos que no progresaban. 
existía el antecedente de alcoholjsmo materno, nos motivó a investigar más profundamente este problema. Ademáa, estudiar la relación existente entre grado de alcoholismo de los padres y desnutrición familiar y también investigar la existencia de retardo mental en los hijos de padrea alcobólicos.

\section{MATERIAL Y METODO}

Se estudiaron 10 niños menores de 6 años que consultaron en la Policlínica de Desnutridos de la Unidad de Nutrición, del Hospital Base de Valdivia, durante el segundo semestre de 1978 y el año 1979 con motivo de presentar desnutrición estacionaria, y en los que además se pesquisó el antecedente de alcoholismo materno. Posteriormente, se amplió el estudio individual hacia el grupo familiar, integrándose hermanos, escolares y adultos. Se conformó de este modo el grupo total de estudio que ascendió a 19 personas. Es importante destacar que algunos de estos nin̄os no se ebcuentran bajo la tutela materna, unos permanecen en hogares de familiares, otros habian sido enviados a CONIN, para su recuperación.

Cada integrante del gapo estudiado fue evaluado con un enfoque multidisciplingrio. El análisis de cada persona incluyó:

1) Evaluacion nutricional: los pacientes estudiados llegaron derivados desde la Unidad de Control de Niño Sano, por haberse pesquisado un déficit nutricional importante, segín norma implantada por el Ministerio de Salud.

Inicialmente se realizó la evaluación nutricional utilizando la clasificación de Gómez que consider los parámetros Peso-Edad. Cada niño fue sometido a todas las acciones del Subprograma de Control de Niño Sano con Déficit Nutricional.

Para la evaluación de estado nutricional actual se utilizaron los patrones antropométricos de Iowa y Boston. Se tomaron los indicadores de Edad-Talla y Peso-Talla. La magnitud del daño nutricional se expresó en número de meses de retardo.

2) Evalución Médico-Pediátrica: a cada grupo familiar se le efectuó anamnesis completa, búsqueda de antecedentes en fichas obstétricas maternas y fichas de morbilidad y Control de Niño Sano de los pacientes. Se realizó examen físico completo y con el fin de facilitar la clasificación del síndrome en grados, se calificaron como leves a los pacientes con menos de cinco signos clínjeos de S.F.A., moderado con cinco a nueve y severos con más de diez rasgos clínjeos.

Se efectuó a cada paciente los siguientes exámenes de laboratorio: Hemograma, Examen de Orina, Radiografía de Carpo y Cráneo, Dermatoglifos, refiriéndolos finalmente a la Unidad de Psicologia de la Universidad Austral.

Dado a que los pacientes no concurrían a los controles para realizar su estudio, se efectuaron visitas médicas domiciliarias. En éstag se realizó: observación del hogar, encuestas alimentarias e incluso anomnesis cuando fue necesario.

3) Evaluacion Psicologica: cada persona fue objeto también de una evaluación psicológica, considerándose los casos con escolaridad y sin ella.

Se inició así la evaluación con una apreciación clinica, del niño, actitud durante la prueba y datos de anamnesis en relación a desarrollo psicomotor. En la investigación del desarrollo psicomotor e intelectual se emplearon las siguientes escalas: para lactantes y preescolares se utilizó la "Escala de Evaluación del Desarrollo Psicomotor" de Soledad Rodríguez y col. (EEPD), con el fin de evaluar un cierto nivel de rendimiento del desarrollo psicomotor del menor y que permite determinar un coeficiente de desarrollo en relación a normalidad, riesgo y retraso de éste.

A los escolares que integraron el estudio se intentó aplicar la Escala de Inteligencia de Wechsler para Niños (Wisc), pero debido a las dificultades que presentaban los menores con sus madrea para concurrir a las citaciones, se decidió utilizar la Escala BinetSimon que entrega una Edad Mental y un Coeficiente Intelectual (CI), y permite una clasificación aproximada e inmediata de la capacidad intelectual.

En relación a los adultos que conformaban el grupo fomiliar, se utilizó la Escala de Inteligencia de Wechsler para adultos (WAIS). Cada paciente fue estudiado en forma secuencial por los profesionales integrantes del equipo multidisciplinario en el siguiente orden: nutricionista, médico, psicóloga. Se realizaron a su vez reuniones periódicas del equipo, con el fin de haces evaluaciones parciales de los resultados.

\section{RESULTADOS}

PRIMER GRUPO FAMILIAR: 5 hermanos, madre bebedora exagerzda desde la edad puberal. Muere accidentalmente a los 32 años de edad por ingestión de ácido nuriático. Primer hija 27 años, ano. pudre de familia. Segundo hijo 25 años, tiene oblo vignos leves de S.F.A., como tella baja y moderada ptoeís palpebral, C.I. de 84 (inteligencia normal lenta). Se consideró sóło como "sospechoso de S.F.A.", escolaridad 4.0 medio, con dificultad de sprendizaje.

Casa clinico N." 1.

24 años, sexo temenino, presenta signos moderados de S.F.A., los enumerădos anteriormente. más hipoplasia malar, asimetría del mentón, palader asimétrico, C.1. 73 R.M. leve y escolaridad 7. ${ }^{\circ} \mathrm{b}$. sico. Casade, I hija normal.

Caca clínico N."2.

20 años, sexo masculino, desteca: talla baja, mierocefaita: $\mathbf{3 1}, 5 \mathrm{cra}$, ptosis palpebral, hịpoplasia malar, palariar ojival, asimetria de pabellones auriculares, pabellones eimples. Tórax en escudo, hipertelia, prognetismo relativo. Se calificó como S.F.A. severo. Agiste a taller encuela epecial.

Caso clínico N. ${ }^{\circ}$.

12 ańos, sexo mequlino, talla $1,24 \mathrm{~m}$, peso $24 \mathrm{~kg}$, bajo percentil 3, microcefalis de $49 \mathrm{~cm}$, rasgos facialé de S.F.A. más acentuados. 
Hipogenitalismo. Se calificó como S.F.A. severo. Rx. eráneo: microcefalia, protrusión facial, dificultad de lenguaje. C.1. 50 R.M. moderado (2." y 3.er caso. Folo 1).

SEGUNDO GRUPO FAMILLAR: madre 30 nños, bebedora deade los 14 atros de edad, hija de pedren alcohólicos; 6 embarazos: 2 partos prematuros, 4 de término. 4 bjjos vivos, dos fallectdos en la primera infancia, procedian de partos premsturos. Hijo mayor 16 años, de especto físico y desarrollo psicomotor normal, trabaja cotno obrero panificador.

Caso elínico N. 4.

13 y medio an̂os, sexo masculino, $33 \mathrm{~kg}$ peso, $1,28 \mathrm{~m}$., bajo el percentil 3 , peso de nacimiento $3,500 \mathrm{~kg}$. Disformogénesis moderada. destaca retardo paberal. C.I. 70 R.M. leve, edad mental 9 años 6 meges. Escoleridad 5." básico.
Caso elínico $\mathrm{N}^{\circ} 5$.

10 años, sexo mesculino, 1,24 m. $25 \mathrm{~kg}$. pego, en percentil 3 . Peøo nacimiento $2,500 \mathrm{~kg}$. Disformogénesis moderada, deateca retardo poberal además de ptosis palpebral, hipoplabia malar, etc. Hipogenilalismo intenso, aberture palpebral $2,5 \mathrm{~cm}$., C.I. $65 \mathrm{~F} . \mathrm{M}$ moderado, edad menial 6 años 8 meaes. Ercolaridad 2. ${ }^{\circ}$ básico.

Caeo elínico N. ${ }^{\circ}$.

5 años, вexo femenino, $12,750 \mathrm{~kg}$., talla $94 \mathrm{~cm}$., bajo percentil 3 , C.C.: 46,5 cm., menos de 2 D.S. Peso nacimiento 2 kg. Disformogénesie scvera, microcefalja. Blefarofimosis: abertura palpebral 2 cm. Hipoplasia malar, prognatiomo relativo. C.L. 64 R.M. moderado. Edad mental 3 años 2 meses. Vive desde los 3 meses de edad con la abuela materna. Al mes de edad peraba 2,100 ke. Hiperactividad.

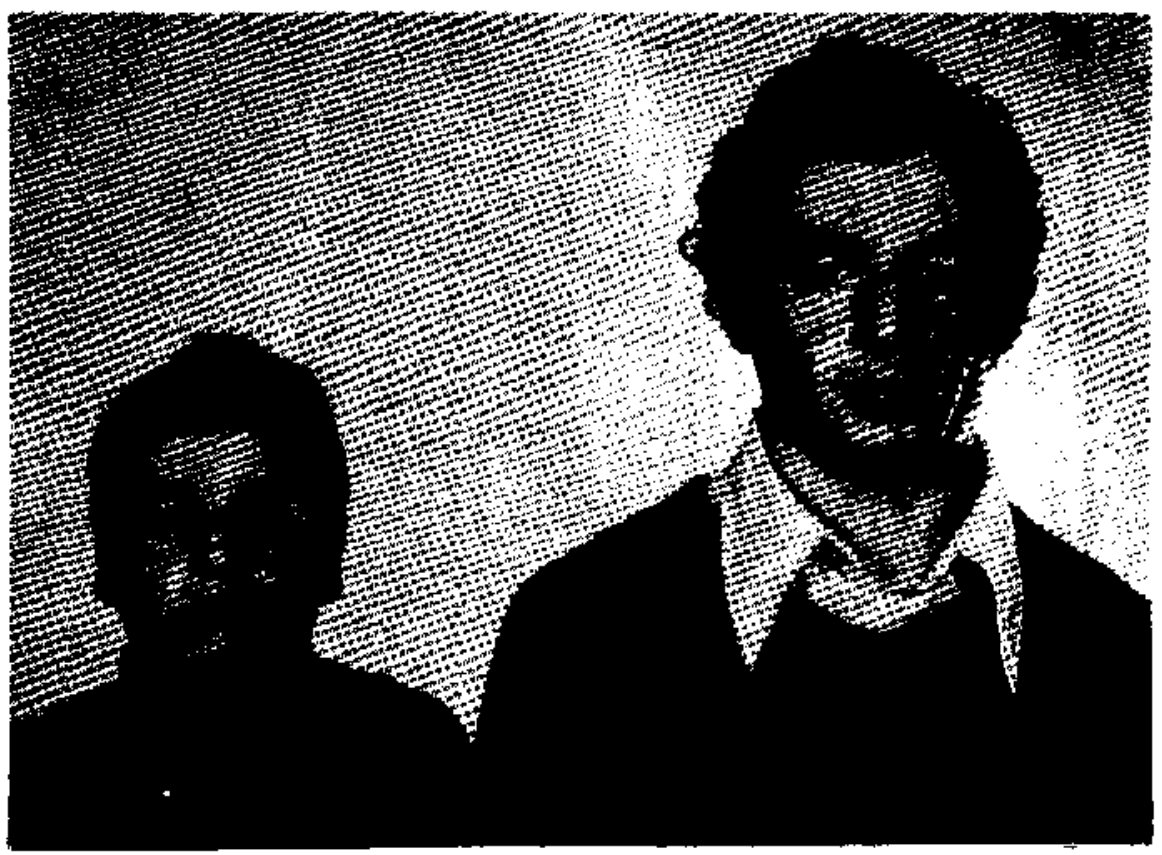

Fifgurm 1. Das bermanos con S.F.A., de 12 y 20 nînos, con talla beja, microcefalia, ptosis pelpebral, hipoplasin maiar.

TERCER GRUPO FAMILIAR: Madre 32 anos, bebedora excesiva desde hace más o menos 14 años, actualmente totalmente alcohólica. 5 embarazos: 4 parto de término, 1 prematuro gemelar. 1 hijo fallecido y 5 hijos vivos. Hijo mayor 11 años, sexo masculino, peso 24 kg., talla 1,20 mt., bajo percenlil 3, peso de nacimiento $2,500 \mathrm{~kg}$. Rasgos leves de S.F.A. C.I. 71 R.M. leve, edod mental 7 años 10 meses. Escolaridad 3. er año básico, dificultad de oprendzaje. Se calificaba como "soвpechoso de S.F.A.".

\section{Camo clinico N.*7.}

9 años 5 meses, aexo masculino, $1,19 \mathrm{~m} .23 \mathrm{~kg}$. Hajo percentil 3, raszos moderados de S.F.A. C.I. 60 R.M. moderado. Edad mental 5 años $B$ meses. No ngiste a la escuela por alter aciones canductuales. Peso nacimiento 2,500 kg. Hiperactividad.

\section{Ceso clínico $\mathbf{2}{ }^{\circ} 8$}

8 eitos 6 meses, sero masculino, peso $21,500 \mathrm{~kg}$., $1,19 \mathrm{mt}$. estatura. en percentil 3, rasgoe moderados de S.F.A., no asiste a la escuela, C.I. 78 R.M. leve, edad ments] 6 años 8 meses. Alteración edad ósea. Peso nacimiento 2,400 kg.

Caso elínico N."9.

3 años 2 meses, sexo femenino (melliza), $8,500 \mathrm{~kg}$., talla $75 \mathrm{~cm}$. bajo percentij $3, \mathrm{C} . \mathrm{C} .=45 \mathrm{~cm}$. Microcefalia, headidura palpebral 2,3 cm., estrabismo convergente. Disformogénesis severa. Coeficiente desarrollo 0,50. Retrago psicumotor. Peso de nacimiento $1,580 \mathrm{~kg}$, talla $38 \mathrm{~cm}$. Incoordinaciôn motora. Hipernctividad

Caso clínico $\mathrm{N}^{\circ} 10$.

3 anos 2 meses, sexo femenino (mellize 2), 9,600 kg., $74 \mathrm{~cm}$. C.C.: $46,5 \mathrm{~cm}$., bajo el percentil 3. Peso as cimiento $1,600 \mathrm{~kg}$. talla $40 \mathrm{~cm}$. Disformogénegis severa, en ambas mellizas exiote un retardo de la edad ógea. Coeficiente de desarrollo 0,53. Retraso psicomotor. Hiperactividad. (Foto 2 ). 


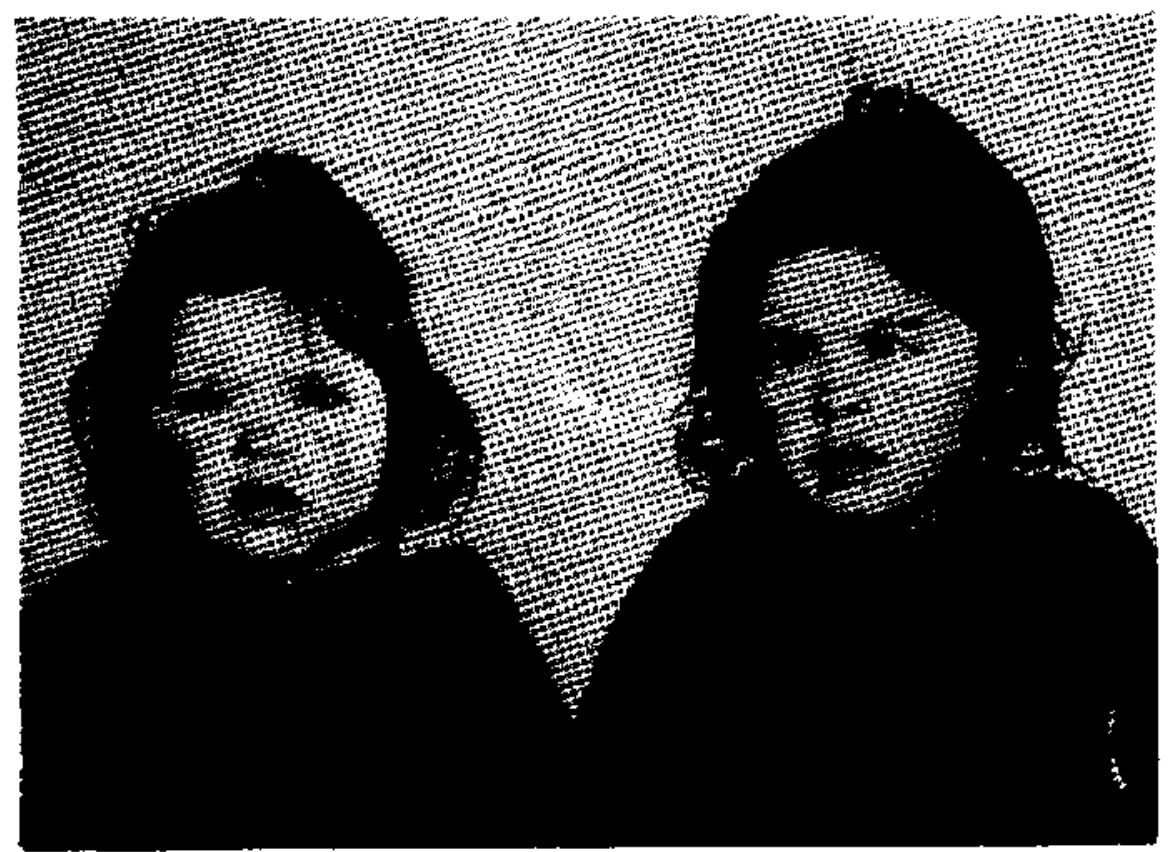

Figura 2. Mellizas de 3 años de edad con S.F.A., destaca: retardo pondoestatural, microcefalia, ptosis palpebral, epicante y esirabiamo convergente (derecha).

CUARTO GRUPO FAMILLAR: madre 37 años, bebedora excesiva desde lo juventud. Abuelos maternos alcohólicos, 3 embarz208, 3 hijog vivos de diferentes padres, ¿abortos? Hijo mayor 12 años, buen desarrollo pondoestatural, $6 .^{\circ}$ sōo bàaico, buen rendimiento.

\section{Caso clínico N. ${ }^{\circ} 11$.}

9 a oos 11 meses sexo femenino, $1 \mathrm{~m} ., 16 \mathrm{~kg}$, bajo el percentil 3. C.C.: 52,5 cm. Pero de nacimiento ${ }^{3} \mathrm{~kg}$.? Camina poco, marcha vacilante, tembloroas, arrofia muacular, no corre. Hipoplasia ma lar, tórax de escudo, hipertelia, pie plano, valgo bilateral, manoe: lineas palmares proximal y diatal, ausencia de línes palmar media. Disformogénesis severa, retardo de la edad ósea. C.I. 38 R.M. severo. Sin escolaridad.

\section{Caso clínico N. 12.}

5 aijos 10 mesce, sexo femenizo, $13,850 \mathrm{~kg}, 103,5 \mathrm{~cm}$. C.C.: $48 \mathrm{~cm}$. microcefalia. Peso de nacimiento 3,200 kg., ptoeis palpebral moderado, aberture palpebral $2,5 \mathrm{~cm}$. Dinformogtnesia modernda, retardo de la edad oeeo. C.I. 102 I.N. promedio, escolaridad kinder con buen rendimiento. Vive con la abuela matema desde lot 6 días de vida

QUINTO GRUPO FAMILlAR: madre 28 años, bebedora exagerada desde hace mís o menos 8 años. 7 embarazos, un aborto espontáneo segundo mes, 3 partos de térnino, 3 prematuros, dos hijos faIlecidos: uno a las $24 \mathrm{hrs}$, de vida y otro al mes por bronconeumonie, ambos de parto prematuro. 4 bijos vivos.

\section{Caso clínico N.* 13.}

5 aťos, sexo femenino, pees $12 \mathrm{~kg}$, talla $91 \mathrm{~cm}$, bajo percentil 3 , C.C. $=46,5$, micrucefalia. Disfornogénesis muderada. Dermatugjfos: líneas pseudosimeanss. Rx. cráneo=hipoplasia medio facial. Edad órea relardsda. C.I. 62 R.M, moderado. Edad mental 3 añoe 2 meses. Peso nacimiento 2,200 kg. Hiperactividar.
Coro slinico N. 14.

3 años 10 meses, sexa masculino, $10,700 \mathrm{~kg}$, $82 \mathrm{~cm}$, hajo pereentil 3, C.C.: 46,5, microcefalia. Disformogénesis moderadas. T. Ĺneas pseudosimianas. Clinodactilia. C.I. 69 R.M. moderado, edad mental 3 nños 2 meses. Peso nacimiento 2,200 kg.

Caso clínieo N." 15 .

2 años 4 meges, sexo masculino, $9 \mathrm{~kg}$. peqo. Talla $72 \mathrm{~cm}$., bajo percenti] 3, C.C.: $44,5 \mathrm{~cm}$., menos de 2 D.S. Disformogénevis maderada. Peao naciniento $1,840 \mathrm{~kg}$. Coeficiente desamollo 0.59 , Retraso pgicomotor.

Chso olínico N. “ 16.

1 meg 20 días, sexo fernenino, $2,600 \mathrm{~kg}$, talla $47,5 \mathrm{~cm}$., bajo percentil 3, C.C.: 34,5, menog 2 D.S. Peso nacimiento 2, $100 \mathrm{~kg}$. Disformogénesia severa. Coeficiente desarrollo 0,54, retraso psicomotor. Todo el grupo familiar tiene edad ósee retrasada.

SEXTO GRUPO FAMILLAR: madre 39 2ñ̄os, 17 años de alcoholismo, 6 embarmos, 3 abortos espontáneos, primer hijo 1,400 kq mortinato, 2 hijos vives.

Caso clínico N." 17.

2 años 10 meses, sexo masculino, peso $8 \mathrm{~kg}$. talla 78,5 cm., bajo percentil 3, C.C.: 43,5 cm. Peso de nacimiento 1,400 kg. Rasgos cl5vicos de S.F.A. severog, microcefalis, blefarofinogis, líneas simisna bilateral. Clinodactilia, triradion T', retarda de la edar ónea. Rx. czáneo: impresiones digitiformes, cráneo pequeño. Coeticiente deserrollo 0,41. Retraso poicomolor. Durante los primeros meses de pide se pesquieó soplo cardíoco, el que desapareció al cuarto mes. Hiperactivided. (Foto 3).

Durante el siguiente embarazo, se abstiene de ingerir alcohol y R.N. pesa $2,400 \mathrm{~kg}$, actualmente tiene 1 año 10 meses, un exarnen físico normal. Coeficiente desarrollo 0,71 , "riesgo psicomotor". 


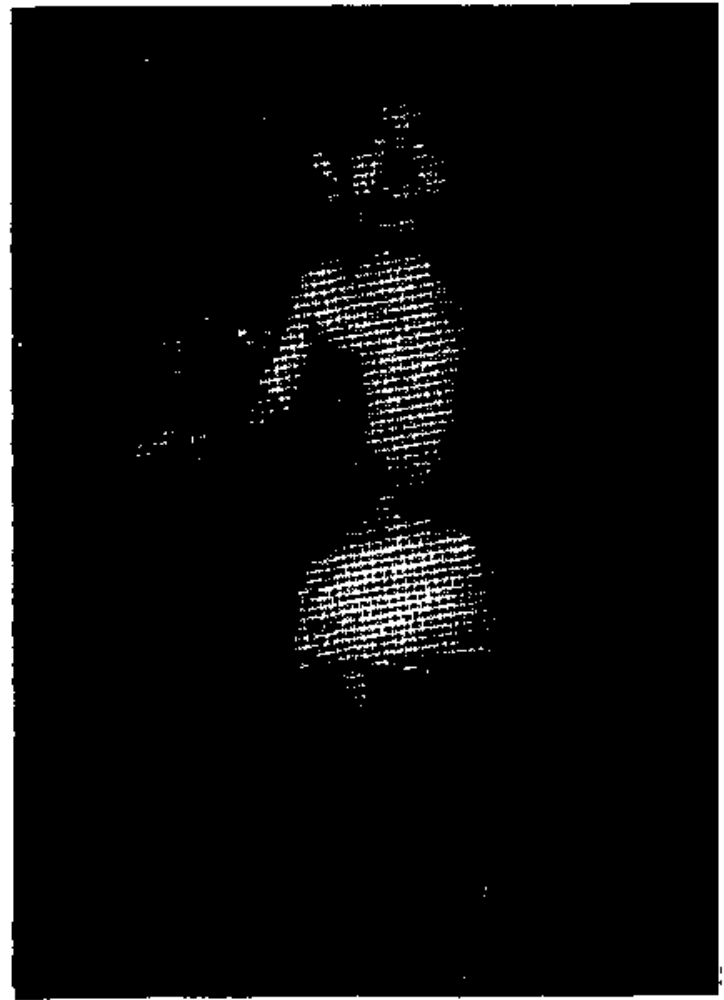

Figara 3.2 año 10 meses, ret ardo pondoestatural bojo el percentil 3. microcefalia, blefarofimosis, epicanto, orejas simples, hipertelia.
SEPTIMO GRUPO FAMILLAR: madre 36 años, bebedora excesjva desde hace 1 años, más de 1 lítro de vino diarianente; 9 embarazos, 7 partos prematuros, todos fallecjeron a los pocos dias de vida. Padre alcohólico. Tiene 2 hijos visos.

Caso clínico $\mathrm{N} .{ }^{ } 18$.

11 años 8 meses, gexo masculino, $1,33 \mathrm{mt}, 28 \mathrm{~kg}$, hajo percentil 3 , C.C.: $50,8 \mathrm{~cm}$., menos 2 D.S. Hipoplasia medio facial canfirmada con Rx. de cránea. Edad ósea 9 años. Peso nacimienta $2,300 \mathrm{~kg}$. S.F.A. severo. Sin escolaridad. C.J. 65. Hiperaclividad.

Cago clinico $N_{+}{ }^{\circ} 19$.

6 añeg, sexo masculino, $17,500 \mathrm{~kg}$ + $103 \mathrm{~cm}$. C.C.; $49,5 \mathrm{~cm}$, microcefalia, abertura palpebral $2,8 \mathrm{~cm}$. Ptosia palpebrai, epicanto, orejas simples. Rx. de cróneo hipoplasia mediu facial. Sin escolaridad. C.I. 50. (Foto 4$)$.

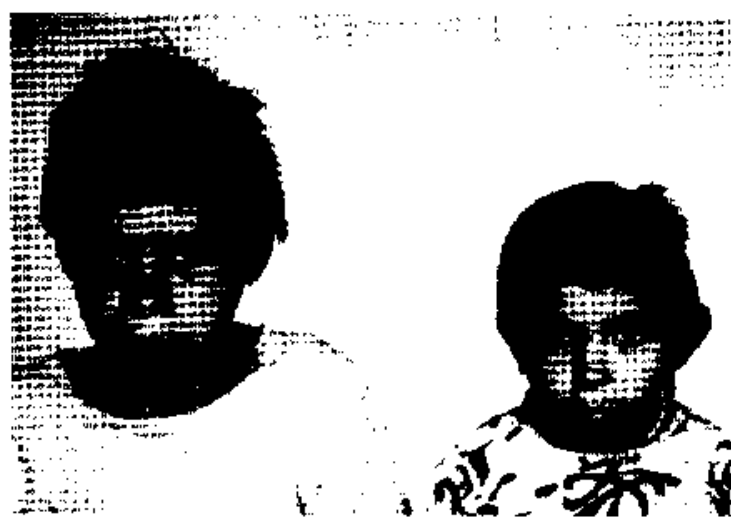

Figura 4. Dos hermanos: microcefalia, orejas simples, labio inferior fino. Hipoplasia medio facial.

Figura 5. Radiografía de cráneo, se observa: hipoplasia medio facial y protrusión marilares.

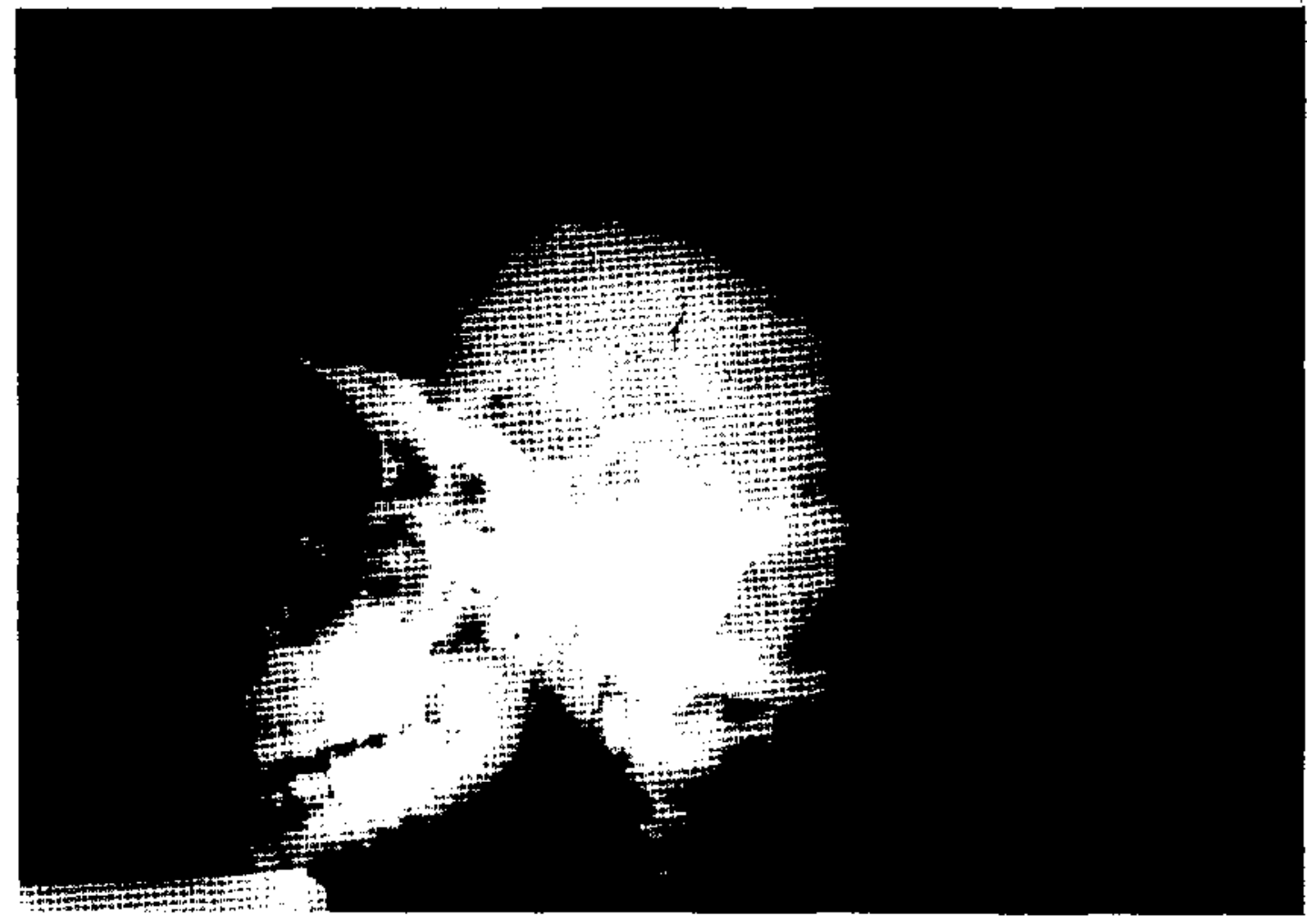


En la Tabla 1 se resumen los rasgos máb relevantes de los 7 grupos familiares.

Se incluyen 3 pacientes que no se contabilizaron como portadores de S.F.A., dos de ellog se calificaron como "sospechosos de S.F.A.", por tener sólo un grado leve del sindrome y un retardo mental leve. El segundo hijo del sexto grupo tiene importancia en su grupo familiar.

En la Tabla 1 observamos que existe una relación directamente proporcional entre grados de S.F.A. y C.I. o desarrollo psicomotor. Se observa que los hijos mayores presentan el síndrome leve o moderado, siendo severo en los menores (grupos familiares 1, 2, 3,5 y 7 ). En los grupos familiares 4 y 6 no se observa esta distribución.

Así en el cuarto grupo familiar, llama la atención la gran variabilidad de fenotipos y de la función intelectual. El hermano mayor es normal, el caso clínico N. ${ }^{\circ} 11$ presenta un S.F.A. y un retardo mental severo, aumentado por falta de escolaridad y de privación materna y ambiental importante. En el caso clínico $\mathrm{N}_{0}$ 12, retirado de su medio durante el período de recién nacido, presenta un S.F.A. moderado y un C.I. normal. Una situación semejante a la descrita es

TABLA NOI- RESULTADOS DE ESTUDIO DE S.FA. HOSPITAL REGIONAL DE VALDYIA $107 \theta-1979$

19 GRUPO FAMILIAR

\begin{tabular}{|c|c|c|c|c|c|c|c|c|}
\hline № & SEXO & EDAD & ESC. & SFA & EX.LAB. & DERMAT. & c.t. & DIAGNOSIICO \\
\hline & M & 26 & $\Delta M$ & Leve & N & $\mathrm{N}$ & 84 & 1.N. Lenta "Sospechoso SFA" \\
\hline 1 & $\mathbf{F}$ & 24 & TS B & Mod. & $M$ & $\mathbf{N}$ & 73 & R.M. LaYe \\
\hline 2 & $m$ & 20 & TEE & Sev. & N & N & 36 & R.M. Severo \\
\hline 3 & $m$ & 12 & E.Esp. & Set. & Alter. & N & 30 & F.M. Modrredo \\
\hline
\end{tabular}

$$
\text { 2E GRUPO }
$$

\begin{tabular}{|c|c|c|c|c|c|c|c|c|}
\hline 6 & $M$ & $13 a 6 m$ & Se日 & Mod. I & $\mathbf{N}$ & $\mathbf{N}$ & 70 & R.M. Leve \\
\hline 5 & $m$ & Da $6 \mathrm{~m}$ & 298 & Suy & N & M & s5 & R.M. Mederado \\
\hline 8 & $F$ & 5 & S/E & ser. & * & $\mathbf{N}$ & 54 & R.M. Moderado \\
\hline
\end{tabular}

3P GRUPO FAMILIAR

\begin{tabular}{|c|c|c|c|c|c|c|c|c|}
\hline Ne & SEXO & EDAD & ESC. & $S F_{A}$ & EX. LAB. & DERMAT. & c.I & DIAGNOSTICO \\
\hline & M & 11 & 3! & Leve & * & N & 31 & R.M. Leve "Sospethone SFA" \\
\hline 7 & M & $905 \mathrm{~m}$ & S/E & Mod & N & N & 60 & R.M Moderado \\
\hline 8 & M & $80 \mathrm{~cm}$ & 19 $\mathrm{B}$ & mod & Alter. & N & 78 & F.M. Leve \\
\hline 9 & $\mathbf{F}$ & 30 $2 \mathrm{~m}$ & SIE & Sevi & Altur. & $\mathbf{N}$ & 0.50 & Rotraso Psicomotor \\
\hline 10 & $\mathbf{F}$ & $302 \mathrm{~m}$ & SIE & Sev. & Alter. & $\mathrm{N}$ & 0.53 & Rotraso Psicomoter \\
\hline
\end{tabular}

tํ GRUPO

\begin{tabular}{|c|c|c|c|c|c|c|c|l|}
\hline 11 & $F$ & $9017 m$ & S/E & Sew & Alter. & Alter. & Jo & R.M. Severo \\
\hline 12 & $F$ & $5010 \mathrm{~m}$ & Kind. & Mod. & Aiter. & $N$ & 102 & I. N. Promedio \\
\hline
\end{tabular}

5E GRUPO FAMHLIAR

\begin{tabular}{|c|c|c|c|c|c|c|c|c|}
\hline $\mathbf{H}^{\mathbf{e}}$ & SEXO & EDAD & ESC. & SFA & $E X, L A B$ & DERMAT. & C I & DIAGMOSIICO \\
\hline 13 & $\mathbf{F}$ & 5 & Kind. & Mod. & Alter. & Allet. & 62 & R.M. Moderado \\
\hline 16 & M & $30 \times \mathrm{m}$ & SIE & Mard. & Altot. & Alter. & 69 & A.M. Moderado \\
\hline 15 & M & $206 \mathrm{~m}$ & SIE & Mad. & Alter. & $\mathbf{N}$ & 0.58 & Retraso Psicomator \\
\hline 15 & $\mathbf{F}$ & 1. 20d: & - & Ser. & Alter. & Alter. & 0.64 & Retraso Paicamotor \\
\hline
\end{tabular}

GE GRUPO

\begin{tabular}{|c|c|c|c|c|c|c|l|l|}
\hline IT & $M$ & $2010 \mathrm{~m}$ & - & Ser & Alter & Aller & O.61 & Retreso Pgitamolor \\
\hline & $M$ & $106 \mathrm{~m}$ & - & - & $\mathrm{N}$ & N & 0.71 & Riesgo Psicomolor \\
\hline
\end{tabular}

79 GRUPO

\begin{tabular}{|c|c|c|c|c|c|c|c|l|}
\hline 10 & M & $108 \mathrm{~m}$ & S/E & SeY & MLter, & N & 65 & A.M. Moderado \\
\hline 19 & M & 60 & S/E & Ser & Altet & Al10r & 50 & A.M. Moderado \\
\hline
\end{tabular}


el caso clínico N.o 6, retirado de su medio a los 3 meses de edad, presenta un S.F.A. severo con retardo mental moderado y peso de nacimiento bajo.

En el sexto grupo familiar, el caso clínico N." 17 , se catalogó como portador de S.F.A. sólo a los 2 años de edad, las hipótesis diagnósticas anteriores eran: Trisomia 18, Blefarofimosis familiar, Cardiopatía congénita. En el siguiente embarazo, la madre se abstiene de ingerir alcohol, el hijo es físicamente normal, pero con riesgo psicomotor, por ausencia de estimulación materna.

En la Tabla N." l-A se resumen todos los rasgos clínicos encontrados en los pacientes y se relaciona la frecuencia de éstos con el grado de severidad del S.F.A.

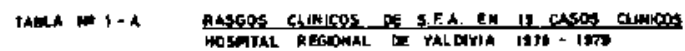

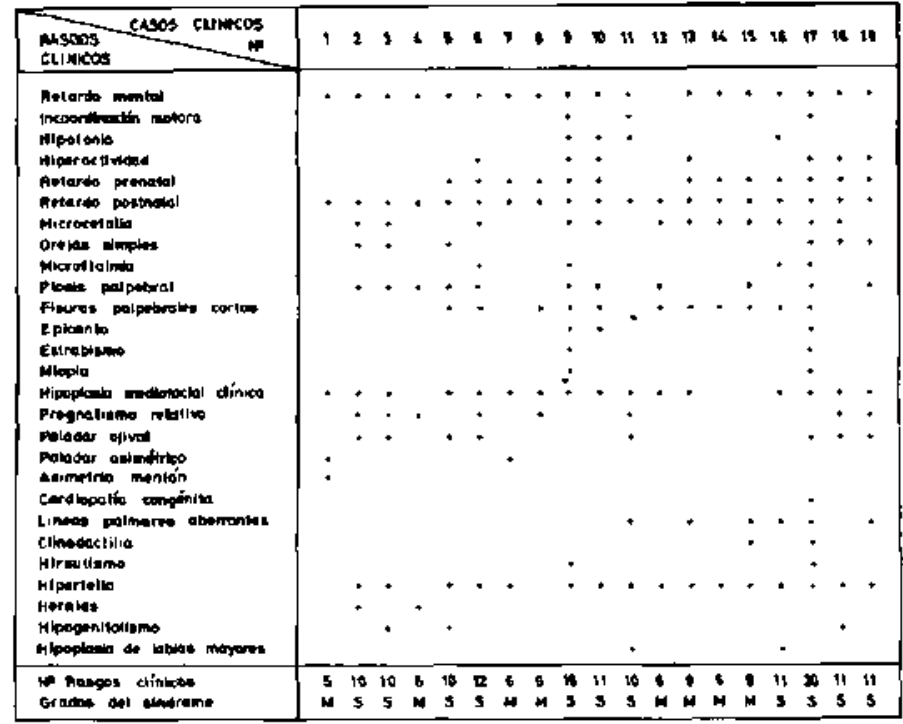

TABLA N*2.- FRECUENCIA DE RASCOS CLINICOS DE S.F.A. EN 19 NINOS HOSPITA AEGIONAL OE WALDIVIA 1970-1979.

\begin{tabular}{|c|c|c|}
\hline AASCOS CLINICOS DE S.F.A. & $H$ DE EASOS & PCRCENTA_E \\
\hline AETAADO MENTAL & $19-19$ & 94,7 \\
\hline INCOOROIHACION MOTCAA & $9-19$ & I S, \\
\hline HIPOTONIA & $4-19$ & 21 \\
\hline HIPERACTIYIOAO & $7-19$ & 36,8 \\
\hline RETARDO DEL DESARROLLO PRENATAL " & $13-10$ & $6,8,4$ \\
\hline RETARDO DEL DESARROLL POSTNATAL & $18-19$ & 100 \\
\hline MICAOCEFALIA & $12=10$ & 63 \\
\hline ORE.JAS SHMPLES & $5-14$ & 31,5 \\
\hline MICROFTALMIA & $4-19$ & 21. \\
\hline PTOSIS PALPEBRAL & $11-19$ & 57,8 \\
\hline FISURAS PALPE BRALES CORTAS & $11-19$ & 57,0 \\
\hline EPICANTO & $3-19$ & 5,7 \\
\hline ESTRABISMO & $2-19$ & 10,5 \\
\hline MIOPIA & $2-19$ & 10,5 \\
\hline HIPOPLASIA MEDIOFACHL CLIWIEA & $16-19$ & 04.2 \\
\hline PROGNATISMO RELATIVO & $8-10$ & 42. \\
\hline PALAOAR OUNAL & $0-10$ & 42. \\
\hline Paladar A SIME TRICO & $2-18$ & 10.5 \\
\hline ASIMETRIA OEL MENTON & $1-19$ & 5,2 \\
\hline CAROHOPATIA CONGENTA & $1-10$ & 5,2 \\
\hline LINEAS PALMARES ABERFANTES & $6-19$ & 31,5 \\
\hline CLINODACTILIA & $2-19$ & 10.5 \\
\hline HIRSUTISMO & $2=10$ & 10,5 \\
\hline HIPERTELIA & $16=19$ & 04.2 \\
\hline HERNIKS & $2-19$ & 10.5 \\
\hline HIPOGEWITALISNO & $3-10$ & 15.7 \\
\hline HIPOPLASIA DE LABIOS MAYORES & $2=19$ & 10,5 \\
\hline
\end{tabular}

* = SE DESCONOCE PESO DE NACIMIENTO EN TRES CASOS CLINICOS
En los 19 pacientes estudiados se observa una frecuencia variable de síntomas y sjgnos, que se describe en la Tabla 2, en ésta podemos observar la distribución de los signos, siendo el de mayor frecuencia el retardo postnatal, seguido por retardo mental, hipoplasia medio facial, hipertelia, retardo prenatal, fisuras palpebrales cortas, ptosis palpebral, etc. Son menos frecuentes cardiopatías congénitas, estrabismo, miopia, etc.

El desarrollo postnatal bajo fue el rasgo clínico que presentaron la totalidad de los pacientes, la magnitud del daño nutricional, de los menores de 6 años, podemos observarla en número de meses, según edad-talla, en la Tabla 3.

En relación a este parámetro cabe destacar que, en los hogares de madre alcohólica visitados, se detectó una importante desorganización del hogar, en especial en la frecuencia y horario de comidas; siendo los resultados de la encuesta alimentaria muy deficientes, sin embargo, el consumo de bebidas alcohólicas era regular y frecuente.

Solamente las madres de los grupos 3,5 y 7 reconocieron ingerir diariamente alcohol en cantidad equivalente a 1 litro o más de vino, sidra o pílsener. 
TABLA NE3.- ESTADO NUTRITIVO ACTUAL DE MENORES DE 6 ANOS CON S.F.A. HOSPITAL REGIONAL OE VALDIVIA 1978-1979.

\begin{tabular}{|c|c|c|c|c|c|c|c|}
\hline No & SEXO & EDAD & TALLA & PESO & $\begin{array}{l}\text { EDAD/TALLA } \\
\text { TA }\end{array}$ & $\begin{array}{l}\text { RETARDO } \\
\text { TALLA }\end{array}$ & $\begin{array}{l}\text { DIAGNOSTICO } \\
\text { PESO-TALLA }\end{array}$ \\
\hline 6 & $F$ & $5 \mathrm{~d} 2 \mathrm{~m}$ & 94. & 12.750 & 30 & 26 meses & D 1 \\
\hline 9 & $\mathrm{~F}$ & 40 & 79 & 9,620 & 10 & $36 " 1$ & NORMAL \\
\hline 10 & $F$ & 40 & 82,5 & 9.500 & $106 \mathrm{~m}$ & $15 "$. & N. LIMITE \\
\hline 11 & $\mathrm{~F}$ & $9 a$ & 100 & $16 .-$ & $4 a$ & 60111 & D 1 \\
\hline 12 & $F$ & $8 a$ & 103. & 14,250 & $4 a$ & $24 " 1$ & DI LIMITE \\
\hline 13 & $F$ & $504 m$ & 91.07 & 12,200 & $206 \mathrm{~m}$ & $34 " \prime$ & D I \\
\hline 14 & $M$ & 4 a $3 m$ & 82. & 10.700 & $106 \mathrm{~m}$ & $33 " n$ & D 1 \\
\hline 15 & $M$ & $2 \mathrm{a} 5 \mathrm{~m}$ & 72,06 & 9.020 & $9 \mathrm{~m}$ & $20 \cdots$ & NORMAL \\
\hline 16 & $\mathbf{F}$ & $2 \mathrm{~m}$ & 48. & 2,660 & $R \mathrm{~N}$ & $" 11$ & 02 \\
\hline 17 & $M$ & $2010 \mathrm{~m}$ & 78.5 & a. - & $10 \quad 4 m$ & $18 " 1$ & D I \\
\hline
\end{tabular}

En los otros cuatro grupos el antecedente fue reportado por maridos, hijos o familiares. En los grupos 5 y 7 el padre también era alcohólico. En 4 familias los abuelos maternos eran alcohólicos. ${ }^{17}$

Todas las madres tienen una historia de alcoholismo de más de 8 a 10 años $y$ dos de 17 años de duración. Sus historias obstétricas, en la mayoría, mostraban abortos espontáneos frecuentes, partos prematuros y mortalidad infantil elevada (grupos 2, 5, 6 y 7).

El retardo mental corno signo fue constante, a excepción del caso clínico N.*12.

El retardo de desarrollo prenatal fue frecuente, excepto en tres casos clínicos y en los tres primeros casos clínicos en que se desconociô el antecedente por haber fallecido la madre.

De los exámenes realizados en este estudio el hemograma estaba normal, en la mayoría de los pacientes, excepto en los que había anemia hipocroma moderada secundaria a una desnutrición primaria.

El examen de orina era normal en todos los pacientes y no se realizó pielografia de eliminación, por no haber signologias, clínico ni de laboratorio, de alteración renal.

La radiografía del carpo reveló edad ósea retardada, de acuerdo al estado nutritivo en los niños con desnutrición primaria ąregada. La radiografía de cráneo mostraba cráneos pequeños con hipoplasia medio facial y protrusión de los maxilares en $6 \mathrm{pa}$ cientes (foto N. ${ }^{\circ}$ 5). En el caso clínico N.o 17 habia además impresiones digitiformes.
Los dermatoglifos revelaron, en 6 casos clínicos, lineas palmares aberrante, como líneas simeana bilateral (caso 17), presencia de dos líneas palmares: la proximal $y$ la distal, con ausencia total o parcial de la línea media (casos clínicos 11 y 19).

\section{COMENTARIO}

El alcohol es teratógeno comprobado2, 3, 4, 5, 6, 7.8,9. $10,11,12,14,15$ y 16 y las alteraciones físicas y poíquicas son muy polimorfas, pues depende de la cuantía y regularidad de la ingesta y de la edad del embarazo, tiene efectos especialmente adversos en los primeros meses del embarazo, en el de sarrollo del sistema nervioso central y dismorfogénesis general. ${ }^{2.5}$

En los hijos de madres alcohólicas, estudiados, se constató un retardo del desarrollo pondoestatural en relación a niños sanos en control, de lo que podríamos deducir que existe una relación directa entre alcoholismo y desnutrición. Lo que no podemos saber es hasta dónde repercute la injuria prenatal y cuánto afecta el ambjente familiar. ${ }^{14}$ Hemos encontrado en la mayoría de los hogares que una parte importante del ingreso farniliar ge invierte en bebidas alcohölicas, no asi en alimentos, a excepción de los hogares de niños que vivían con familiares no alcohólicos.

Por lo tanto, cabría hacerse la pregunta: ¿cuántos de los desnutridos en control, que no progresan, tienen esta misma etiologia?

Encontramos una relación directa entre mayor se- 
veridad de S.F.A. y mayor compromiso del desarrolo psicomotor del paciente, y/o mayor alteración de su nivel intelectual. No obstante el C.I. fue variable con fenotipos semejantes. ${ }^{15}$ Sin embargo, hay que tener presente que un seguimiento de 23 mujeres embarazadas bebedoras, $44 \%$ de los niños seguidos tenian un setardo mental moderado, a los 7 años, y sólo $32 \%$ tenían múltiples rasgos de S.F.A. Lo que indica que en algunos niños el problema de estructura cerebral y/o función puede ocurrir, como "única aparente anormalidad", en la exposición uterina al alcohol. ${ }^{2}$

En la interferencia del desarrollo psicomotor, junto al menoscabo físico, hemos constatado en la mayoría de los casos una deprivación materna importante, determinada por el alcoholismo materno y de otros familiares adultos del grupo. ${ }^{14}$

Los fenotipos encontrados en los pacientes mostraron una severidad creciente, a medida que aumentó el grado de alcoholismo materno. ${ }^{11}$ ( mayor tiempo de metabolización del alcobol?); salvo casos excepcionales en que entraron a jugar otras variables, como menor consumo de alcobol en algunos embarazos a cambio de ambiente de los pacientes.

En cuanto a tiempo de metabolización de alcohol, es importante recordar que la severidad de las alteraciones morfológicas están más relacionadas con los niveles de alcohol no metabolizados, que con los productos metabólicos. ${ }^{6}$ Se ha demostrado que el alcohol difunde rápidamente de la sangre a los tejidos, en forma uniforme, y se le encuentra en las glándulas genitales y esperma, liquido amniótico y sangre fetal en una concentración igual a la de la sangre materna. Están en discusión el mecanismo de acción distrófico y teratógeno; pero se concluye que las anomalías del S.F.A. son producidas por causas complejas, en las que la impregnación alcohólica de] feto, durante el embarazo, se asocia a graves transtornos nutritivos por alteraciones uterinas y perturbaciones alimentarias y metabólicas de la madre. ${ }^{19}$

Por lo tanto, la patogenia no está totalmente establecida. En humanos se demostró que había alteración de enzimas microsomales hepáticas por el alcohol. Esto llevó a Waltman a especular que la ingesta de alcohol, por parte de las madres, afectaría las enzimas fetales, metabolismo de drogas y sustrato esteroidal. En un trabajo de 2 años, Ulleland observó que el $41 \%$ de los recién nacidos eran pequeños para la edad gestacional y Haggard demostró que las madres alcohólicas tenían el doble de mortalidad infantil. Lo que esta de acuerdo con nuestras historias clínicas obstétricas y mortalidad infantil.

A diferencia de lo comunicado en trabajos al respecto, hemos observado que en algunos casos, al ser reubicados precozmente, los nínos (R.N.) alcanzaron un desarrollo global más aproximado al normal.

En un reportaje de 20 pacientes, en que se relacio- nó dismorfogénesis de S.F.A. con inteligencia, se encontró que el promedio tenía C.I. de 65 y hubo tres S.F.A. con C.I. normales. ${ }^{15}$

En los casos clinicos, en que fue retirado el teratógeno durante el embarazo, el producto concebido resultó físicamente normal. Sin embargo, en la evaluación del desarrollo psicomotor la calificación fue riesgo psicomotor, por efecto de la deprivación materna y ambiental. ${ }^{21}$

La frecuencia de los gignos y síntomas observados en los pacientes (Tabla 2), concuerda con estudios practicados por Sterling y col. en 245 pacientes con S.F.A. y los de Hanson, en 41 pacientes y otros autores.6. 10, 11, 14

Otros estudios realizados han demostrado que la funciốn hipota]ámica pituitaria está normal, de manera que la edad ógea es mayor que la edad-talla, lo que indica que el niño es genéticamente bajo. ${ }^{13} \mathrm{~A}$ diferencia de nuestros niños en que la edad osea está muy atrasada respecto a la edad cronológica y coincide con la edad-talla, de acuerdo a la desnutrición primaria agociada.

En observaciones realizadas en 200 pacientes con S.F.A., 6 tenían anomalías renales y la más frecuente era la hipoplasia renal: incluso se concluye que en pacientes con esta anomalía, debería investigarse S.F.A. e historia materna de alcoholismo. ${ }^{12}$ Lemoin, en 127 S.F.A., sólo uno presentaba malformación renal. ${ }^{19}$ En nuestros pacientes no hubo signologia de patología renal.

E] diagnóstico diferencial debe plantearse con los siguientes síndromes: Trisomia 18, Blefarofimosis familiar, Comelja de Lange, Smith-Lemli Opjtz, Sindrome de Noonan, Síndrome fetal por hidantoína. ${ }^{30,14,15,16}$

Pensamos que los conceptos vertidos en este estudiq deberían difundirse dado que es un problema no solamente de índole médico, sino también social, teniendo por esta razón una importancia incalculable la prevención de este síndrome. Pues según estadísticas nacionales, el $14 \%$ de la población, mayor de 15 años, es bebedora exc esiva y de éstos, uno es de sexo femenino. El hábito se adquiere en la segunda década de la vida, que es el período de mayor procreación.

Si con estos antecedentes aplicamos en nuestro país la frecuencia de S.F.A. observada en Europa y EE.UU. ${ }^{14}$, el problema se agrava, y más aún si se considera que en una publicación ${ }^{19}$ de 127 casos de S.F.A., que procedían de 69 familias, en 15 de éstos el alcoholismo era solamente paterno, en 25 materno y en 29 de ambos progenitores.

Finalmente ante la magnitud de la deprivación materna y en general, ambiental fatniliar, en la mayoria de los grupos estudiadog se considera importante la reubicación de los niños en hogares de familiares, o de menores y por supuesto, intentar tratamiento antialcohólico de los afectados. 
El presente estudio es la primera parte de la investigación sobre S.F.A. pues se continúa con el estudio de nuevos pacientes.

\section{RESUMEN}

Se estudiaron 19 pacientes con S.F.A., hijos de madres alcohólicas y que provenían de 7 grupos familiares.

Se realizó examen clínico, de laboratorio con hemograma, examen de orina, radiografías de carpo y cráneo, dermatodifos y estudio de desarrollo peicomotor $\mathrm{e}$ intelectual.

Como todo teratógeno, el alcohol produjo dismorfogénesis variables según cuantís y regularidad de ingesta o edad del embarazo.

Las alteraciones morfogénicas de S.F.A. estaban en proporción directa con el retardo psicomotor e intelectual.

La severidad del S.F.A. era superior a mayor tiempo de evolución del alcoholismo matemo.

La intensidad del alcobolismo materno o familiar era proporcional a la desnutrición infantil.

Las historias obstétricas de las madres alcohólicas revelaban aumento de la frecuencia de abortos espontáneos, partos prematuros, mortinatos y mortalidad infantil.

\section{REFERENCIAS}

${ }^{1}$ Beukelder, M.D.: Rengl anomalies in the fetal alcohol syndrome. J. Pediatr., 91: 759, 1977.
${ }^{2}$ Clarren, S.K.: Brain malformstione related to prenatal exposure to ethanol. J. Pediatr., 92: 64-67, 1978.

${ }^{3}$ Chistoffel, K. Fetal alcobol ayndrome indizycotic twins. J. Pediatr., 87: 963-967, 1975.

${ }^{4}$ Cazi, G.H.; Altered bex radia in fetal alcahol oyndrome. Lancet 2(7975) 42, 1976.

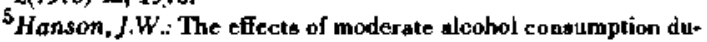
zing pregancy on fetal growth morphogenesis. J. Pediatr., 92: 457-460, 1978 .

${ }^{6}$ Harson, J.W.; Fetal alcobol syndrome. Experience with 41 patients. JAMA 235(14): 1458-1460, 1976.

${ }^{7}$ Jones, K.L.i Paterns of malformations in offspring of chronic alcoholic mothers. Lancet 1: 1267-1271, 1973.

8 Jones, K.L.: Recognition of the fetal alcohol syndrome in early infancy. Lencet 2: 999-1001, 1973.

${ }^{9}$ Kronick, J.8.: Teratogemic effects of ethyl alcohol adrniniatred to pregrancy mice. Am. J. Obst. Gynecol, 124; 676-680. 1976.

${ }^{10}$ Mulvihill, J.J.: Fetal alcohol syndrome: seven new casea. Am. J. Obet. Gynecol. 127(5): 937-941, 1976.

Il Palmer, R.H.i Congenital malformations on offopring of chronic alcoholic mother. Pediarrice 53: 490-494, 1934.

${ }^{12}$ Qaxi, $Q$.: Renal anomalies in fetel alcohol syndrome. Pediatrica 63: 886-889, 1979.

${ }^{13}$ Root, A.: Hypothalamic-pituitary function in the fetal alcohol syndrome. J. Pediatr. 7: 585-588, 1975.

${ }^{14}$ Sterling, H.C.: The fetal alcohol Byndrome England. J. Med. 29: 1963-1967, 1978.

${ }^{15}$ Steissguth, $\boldsymbol{A P}$.: Intelligence, behavior and dysmorphogenesis in the fetal alcohol syndrome: a report on 20 patient. J. Pectiatr. 92: 363-367, $197 \mathrm{~B}$.

${ }^{16}$ Brith. Med. J. 2(6049): 1404-1405, 1976 (Editorial). The felal alcohol syadratne.

${ }^{17}$ Spalt, L.M.D.: Alcoholism evidence of an $\mathrm{X}$-linked recessive genelic characteriatic. JAMA 24l(23): 2542-2544, 1979.

${ }^{18}$ Smith, D.MD.: Fetal alcohol syndrome. Recognizable palterna of human nuelformation. 336-337, 1976.

${ }^{19}$ Lemoine, $P$. Le enfants de parents alcoboliques. Anomalies observées. A propos de 127 cas. Quest Médical 476-482. 25 marzo 1968. 